\title{
ABOUT THE EXPERIMENTAL INVESTIGATION OF RESIDUAL BEARING CAPACITY OF DAMAGED REINFORCED CONCRETE BEAMS INCLINED SECTIONS
}

Klymenko Ye.V., Doctor of Engineering Science, Professor, klimenkoew57@gmail.com, ORCID: 0000-0002-4502-8504

Boiadzhi A.O., $\mathrm{PhD}$, Senior teacher, Polianskyi K.V., post-graduate student Odesa State Academy of Civil Engineering and Architecture kostyapolyanski@gmail.com

\begin{abstract}
The article reports on the results of the experimental studies to determine the effect of concrete damages in compressed zone of rectangular cross-section reinforced concrete beams on their residual bearing capacity of inclined sections. The special experimental installation and hydraulic jack-screw were used for the tests. According to the experimental plan, 16 singlespan freely supported experimental samples of reinforced concrete beams were tested. All examples had own number from Б0 to Б15 (example Б0 was the twin of example 51). The acted load on the samples was applied as a concentrated force and was added in uniform steps with an exposure in $10-15$ minutes. The dimensions of the beams were $100 \times 200 \times 1200 \mathrm{~mm}$ and the working span is $1000 \mathrm{~mm}$. In the beams the artificial damages of the compressed zone of concrete are laid in advance in different sizes with different angles relative to the edge of the beam, and the different size of the shear span $c(1 d, 2 d$, and $3 d)$ are also used. Materials used for beams are: concrete grade C25/30; working longitudinal reinforcement $-\varnothing 18 \mathrm{~mm}$ of grade A500C; constructive longitudinal reinforcement and transverse reinforcement in the form of vertical links $-\varnothing 6 \mathrm{~mm}$ of grade A240C. According to actual codes the physical-mechanical properties materials were determined on the control samples - cubes, prisms and rebars. All samples were destroyed along an inclined section. It has been established that an increase in the shear span (from $1 d$ to $3 d$ ) leads significantly to a decrease in the carrying capacity (especially from $1 d$ to $2 d$ ). As well, within the shear span, the appearance of damage and an increase in their size results in a decrease in the carrying capacity of the samples. The experimental data obtained will be used in the development of a method for determining the residual bearing capacity of inclined sections.
\end{abstract}

Keywords: experimental investigations, reinforced concrete beams, damages in reinforced concrete, destruction, inclined section, shear span, residual bearing capacity.

Introduction. In the current economic conditions, the vast amount of concrete structures operate with a considerable period of life in our country. Unfortunately, during its operation concrete structures, definitely undergo deterioration and/or damages, cracking, corrosion of reinforcement, concrete corrosion, mechanical damages (such as chipped concrete), etc. [1]. The existing national building codes $[2,3]$ there are no instructions or guidance on determining residual bearing capacity of damaged concrete structures that's why important theoretical and experimental research and development of methods for its determination are raised.

Analysis of recent research and publications. At the Odessa State Academy of Civil Engineering and Architecture the residual bearing capacity of concrete elements was studied by Klimenko Ye. V. and his students [4-6 and others] and the results of these studies suggested a number of methods to determine residual bearing capacity of damaged concrete columns, beams for normal sections, etc., but studies of residual bearing capacity of damaged beams inclined sections in the compressed area are not carried out and there is no available scientific information in sources.

The aim of the work. To give results of experimental studies of residual bearing capacity of inclined sections damaged area in compressed reinforced concrete beams.

Research Methodology. Experimental research of reinforced concrete test samples was made 
on a special installation and hydraulic jacks. Beams are designed in such a way that the destruction occurred on inclined sections. Loading was put in uniform degrees, 10-15 minutes of exposure to a concentrated force within a given span of cut. Indications made at the beginning and end of each level loads.

The main part. At the Department of Building Structures at the Odessa State Academy of Construction and Architecture experimental studies were conducted to determine the residual bearing capacity of inclined rectangular sections of reinforced concrete beams with damaged precompressed zones.

16 one-span reinforced concrete beams in sizes $100 \times 200 \times 1200 \mathrm{~mm}$ with working span $1000 \mathrm{~mm}$ were tested. For a sample with a code 51 twin sample with a code 50 was manufactured and tested. For control samples concrete cubes and prisms according to [7, 8] were identified concrete grade used for samples - C25/30; control samples for reinforcing bars according to [9] used was determined longitudinal working rebars $-\varnothing 18 \mathrm{~mm}$ of grade A500C, mounting longitudinal and transverse reinforcement in the form of vertical links - $\varnothing 6 \mathrm{~mm}$ of grade A240C. Sectional beams with dimensions of damages and values of shear span on codes samples are shown in the table. 1.

The tests of beams were made on the universal power plant using hydraulic jacks $Д \Gamma-50$. Research beams tempted as one-span free supported beams exposed to bend under concentrated load. Jack ДГ-50 forms a concentrated force that is controlled by a special pressure gauge of pump station jack and passed on prototypes of reinforced concrete beams. Installation scheme shown in Fig. 1.

According to destruction criterion of beams one of the criteria was made: significant deformation of concrete and rebar, the excessive width of opening cracks, excessive beam deflection and a significant decline and subsequent inability to maintain pressure gauge needle shows. All prototypes were destroyed on an inclined section. Results recorded breaking stress are given in Table. 2. Breaking load and lateral force in the experimental samples are shown in Fig. 2.

According to the experimental data it can be seen that increasing the span cut $c$ (from $1 d$ to $3 d$ ) leads to reducing the bearing capacity in the samples, as the authors confirm [10-12]. Also, the data show relative decrease in residual bearing capacity of the formation within each cut and increase of damage volume. The data reveal addiction, providing the opportunity to develop proposals for the calculation of damaged beams.

Table 1 - Sectional beams with dimensions of damages and values of shear span

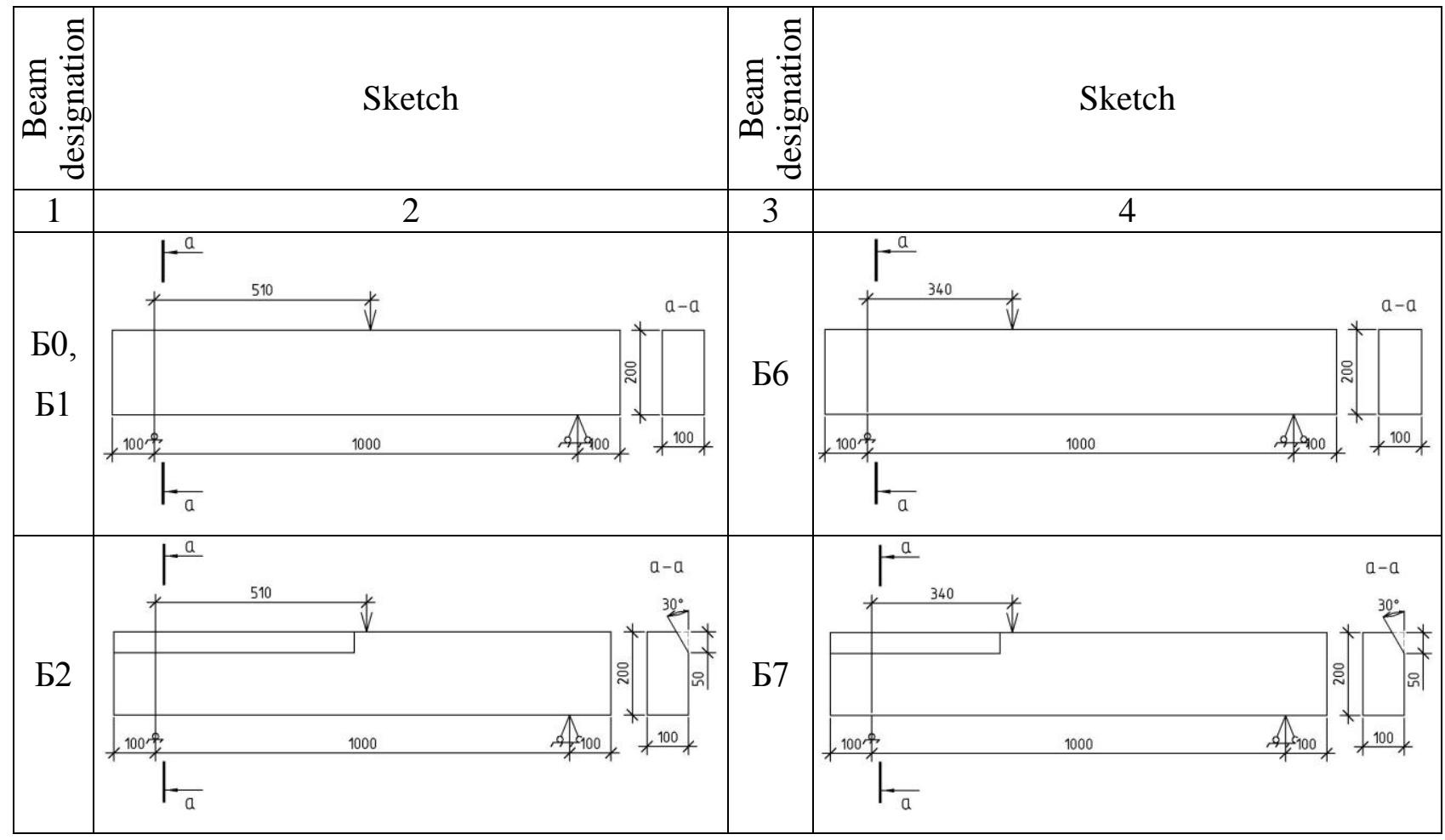


Continue of table 1

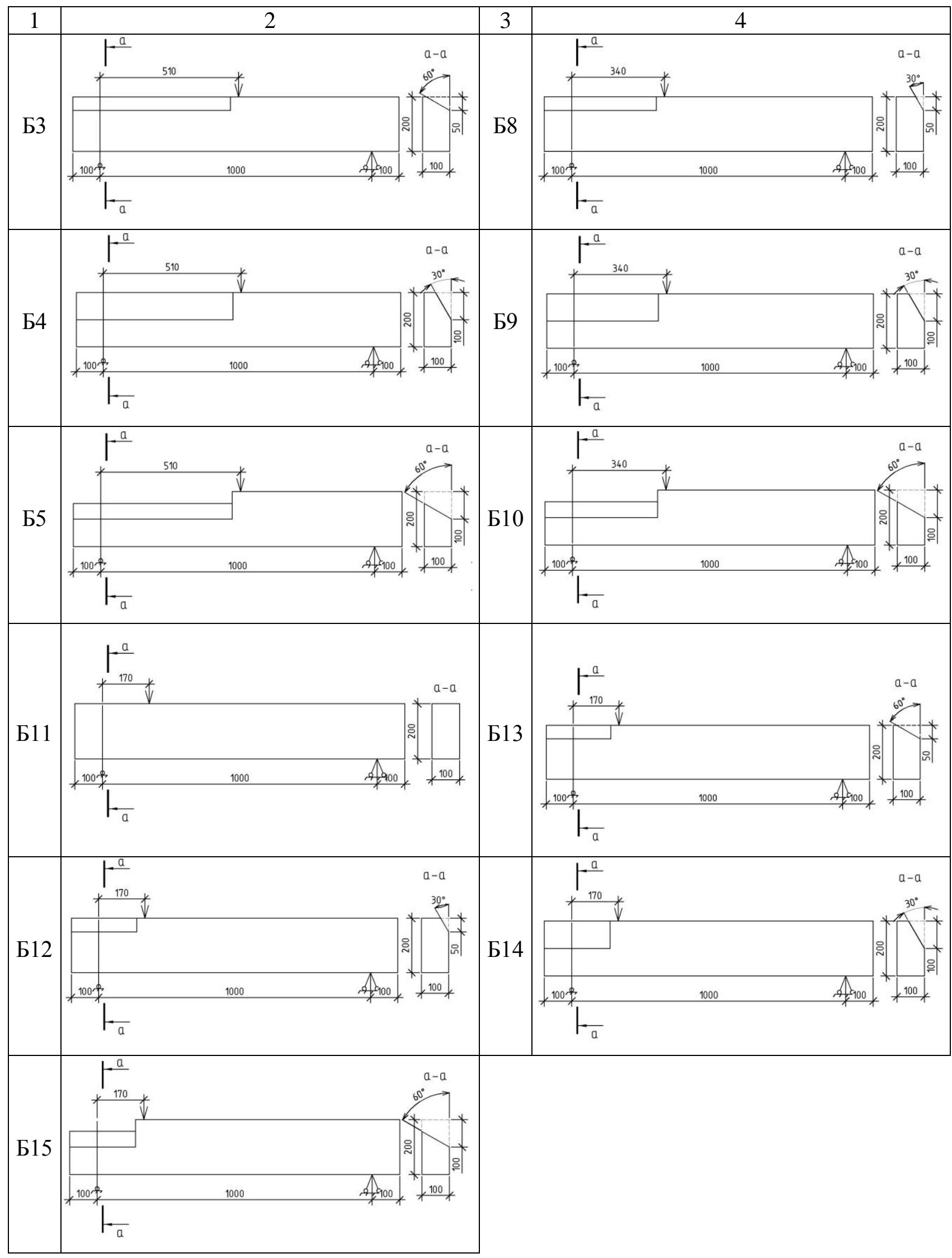




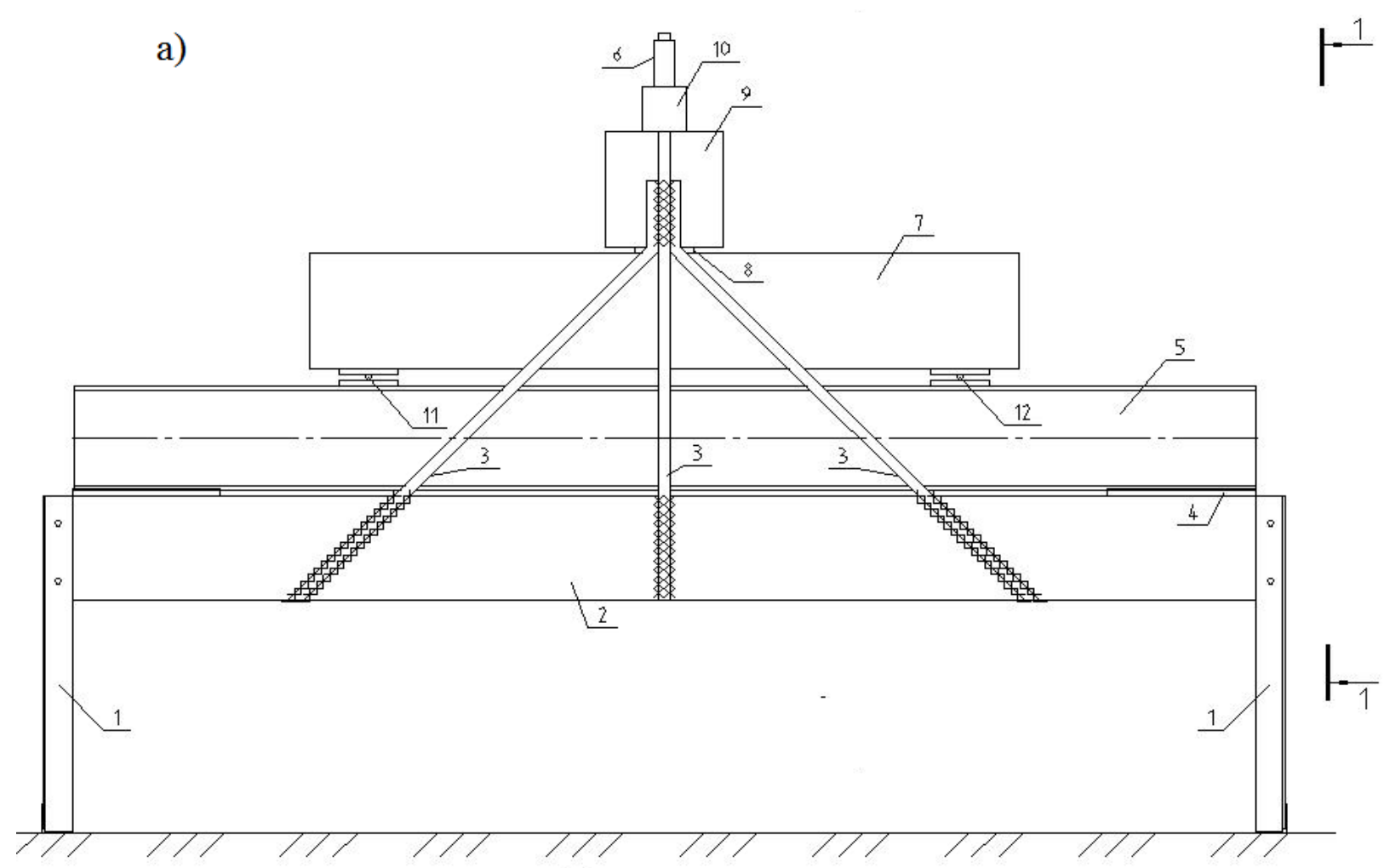

б)

$1-1$

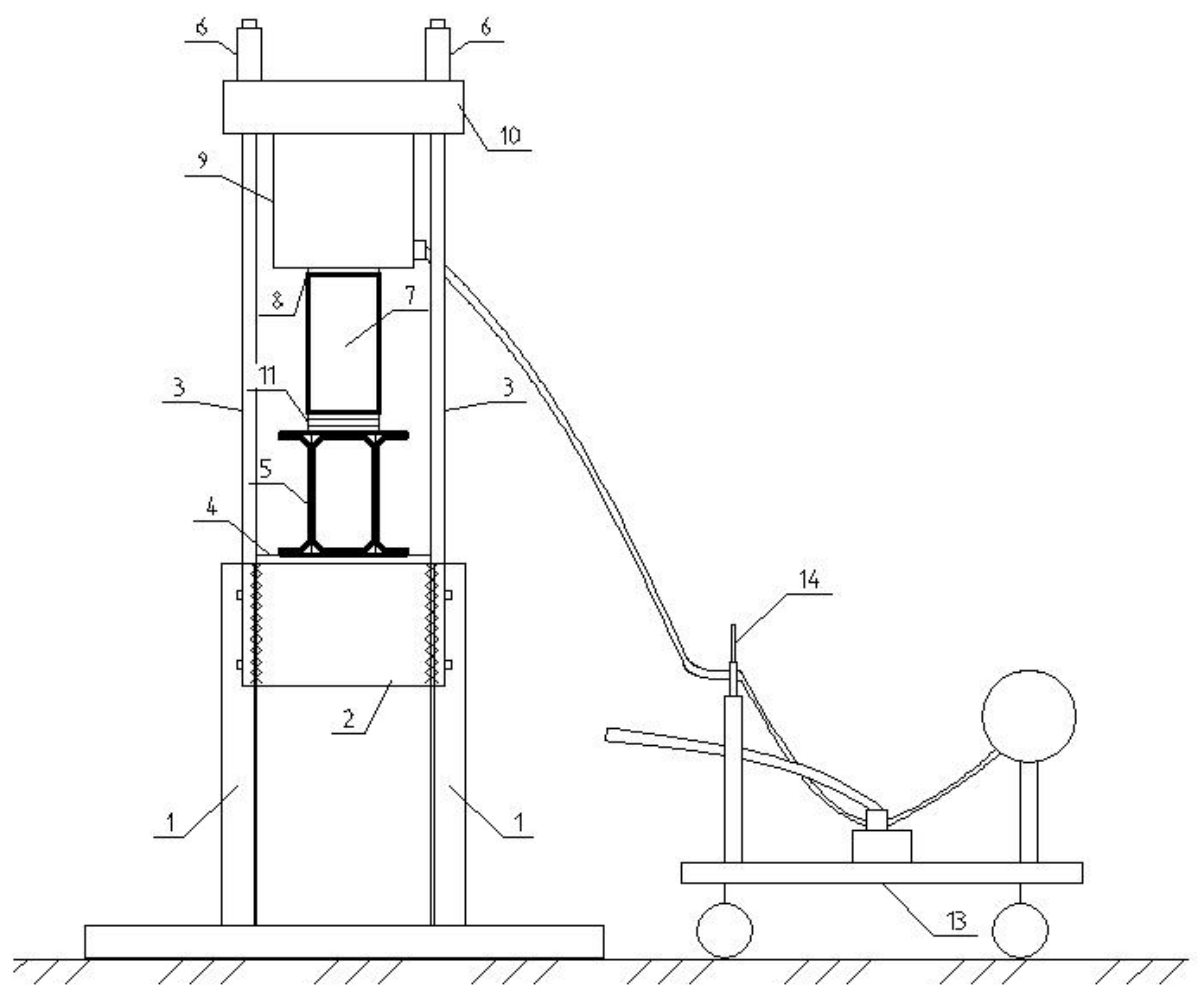

Fig. 1. Installation scheme: $\mathrm{a}$ - front view; 6 - side view;

1 - support; 2 - steel box; 3 - steel bar; 4 - distribution plate; 5 - distribution cross-arm; 6 -screw; 7 - experimental sample; 8 - distribution liner; 9 - jack $D G$-50; 10 - jumper; 11 -support; 12 - support; 13 - pumping station; 14 - pressure gauge 
Table 2 - Maximum fixed loads and transverse forces in experimental samples

\begin{tabular}{|c|c|c|c|c|c|c|c|c|}
\hline $\begin{array}{c}\text { Beam } \\
\text { designation }\end{array}$ & $F_{u}, \mathrm{\kappa N}$ & $V_{u}, \mathrm{\kappa N}$ & $\begin{array}{c}\text { Beam } \\
\text { designation }\end{array}$ & $F_{u}, \mathrm{\kappa N}$ & $V_{u}, \mathrm{\kappa N}$ & $\begin{array}{c}\text { Beam } \\
\text { designation }\end{array}$ & $F_{u}, \mathrm{\kappa N}$ & $V_{u}, \mathrm{\kappa N}$ \\
\hline 1 & 2 & 3 & 4 & 5 & 6 & 7 & 8 & \\
\hline Б0, Б1 & 121,62 & 59,59 & Б6 & 133,28 & 87,96 & Б11 & 158,27 & 131,36 \\
\hline Б2 & 116,62 & 57,14 & Б7 & 99,96 & 65,97 & Б12 & 149,94 & 124,45 \\
\hline Б3 & 98,29 & 48,16 & Б8 & 93,296 & 61,58 & Б13 & 139,944 & 116,15 \\
\hline Б4 & 96,63 & 47,35 & Б9 & 73,304 & 48,38 & Б14 & 124,95 & 103,71 \\
\hline Б5 & 49,9 & 24,49 & Б10 & 56,644 & 37,39 & Б15 & 106,624 & 88,5 \\
\hline
\end{tabular}

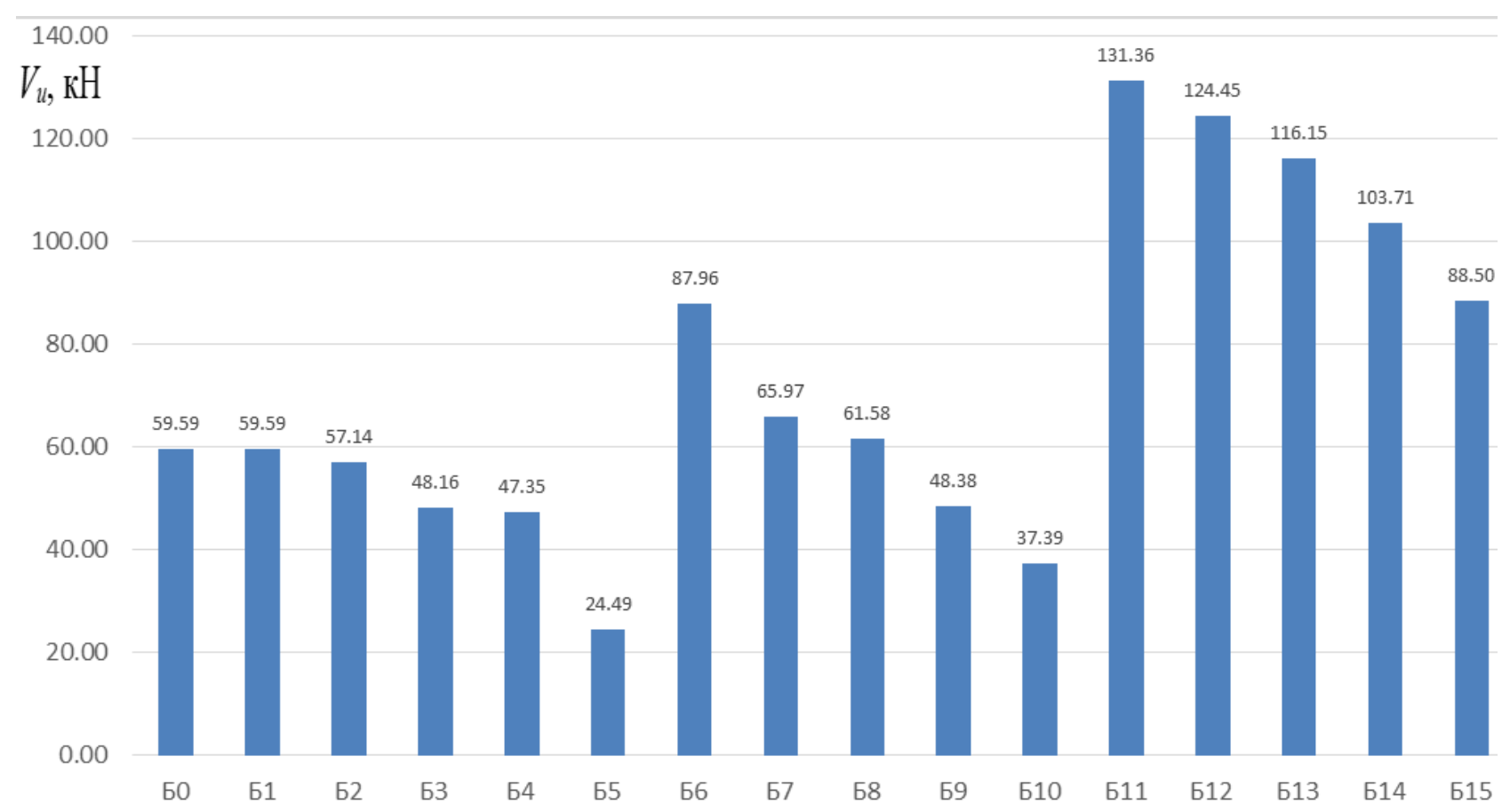

Fig. 2. Ultimate transverse forces $V u$ in experimental samples Б0-Б15

\section{Key findings:}

1. Experimental study of determining the residual bearing capacity of inclined sections for damaged reinforced concrete beams of rectangular section and the data breaking stress in samples.

2. The results of experimental studies have found that within a span of cut formation damages and increase their volume decrease resulting samples bearing capacity and increase a span of cut $c$ (from $1 d$ to $3 d$ ) leads to a reduction of the carrying capacity (especially $1 d$ to $2 d$ ).

3. Operating with received data we are planning to submit proposals regarding the calculation of residual bearing capacity of damaged reinforced concrete beams inclined sections of rectangular section.

\section{References}

[1] Ye.V. Klimenko, Tehnichnyi stan budivel ta sporud, Odessa: ODABA, 2010.

[2] Konstruktsii budynkiv i sporud. Betonni ta zalizobetonni konstruktsii. Osnovni polozhennia proektuvannia: DBN V.2.6-98:2009. K. : Minrehionbud Ukrainy, 2009.

[3] Konstruktsii budynkiv i sporud. Betonni ta zalizobetonni konstruktsii z vazhkoho betonu. Pravyla proektuvannia: DSTU B.V.2.6.-156:2010. K.: Minrehionbud Ukrainy, 2010. 
[4] Ye.V. Klimenko, "Vlyianie povrezhdennosti na prochnost i deformativnost izghibaemykh zhelezobetonnykh elementov", Visnyk Odeskoi derzhavnoi akademii budivnytstva ta arkhitektury, Vol. 46, pp. 175-180, 2012.

[5] Ye. Klymenko, Arez Mohammed Ismael, Capacity of damaged reinforced concrete beams : Monograph. Odessa, OSACEA, 2017.

[6] E.V. Klimenko, T.A. Krutko, Rabota povrezhdennyih zhelezobetonnyih kolonn, Odesa: Odeska derzhavna akademiia budivnytstva ta arkhitektury, 2014.

[7] Betony. Metody vyznachennia mitsnosti za kontrolnymy zrazkamy. DSTU B.V.2.7-214: 2009. K.: Minrehionbud Ukrainy, 2010.

[8] Metody vyznachennia pryzovoi mitsnosti, modulia pruzhnosti i koefitsiienta Puassona. DSTU B V.2.7-217:2009. K.:Minbudrehion Ukrainy, 2010.

[9] Stal armaturnaya. Metodyi ispyitaniya na rastyazhenie: GOST 12004-81. M.: NIIZhB, 1981.

[10] A. S. Zalesov, Yu. A. Klimov, Prochnost zhelezobetonnyih konstruktsiy pri deystvii poperechnyih sil. K.: Budivelnik, 1989.

[11] F.R. Karpyuk, "Mitsnist, trishchynostiitsist ta deformatyvnist pryopornykh dilianok poperedno napruzhenykh tavrovykh zalizobetonnykh elementiv", author's Cand. Tech. Sciences: 05.23.01, Odessa, 2010.

[12] V.M. Karpyuk, "Rozrakhunkovi modeli prohinnykh zalizobetonnykh konstruktsii pry skladnomu napruzheno-deformovanomu stani pryopornykh dilianok", diss. Cand. Tech. Sciences: 05.23.01, Odessa, 2012.

\title{
ПРО РЕЗУЛЬТАТИ ЕКСПЕРИМЕНТАЛЬНОГО ДОСЛІДЖЕННЯ ЗАЛИШКОВОЇ НЕСУЧОЇ ЗДАТНОСТІ ПОХИЛИХ ПЕРЕРІЗІВ ПОШКОДЖЕНИХ ЗАЛІЗОБЕТОННИХ БАЛОК
}

\author{
Клименко Є. В., д.Т.н., професор, \\ klimenkoew57@gmail.com, ORCID: 0000000245028504 \\ Бояджі А. О., к.т.н., ст. викладач, \\ Полянський К. В., аспірант \\ Одеська державна академія будівництва та архітектури \\ kostyapolyanski@gmail.com
}

\begin{abstract}
Анотація. У статті представлені результати проведених експериментальних досліджень по визначенню впливу пошкоджень стиснутої зони бетону на залишкову несучу здатність похилих перерізів залізобетонних балок прямокутного перерізу. Для проведення експерименту була використана універсальна силова установка і гідравлічний домкрат. Відповідно до плану експерименту, було випробувано 16 однопрогонових вільно опертих експериментальних зразків-балок. Всі зразки мали свій шифр від Б0 до Б15 (зразок Б0 двійник зразка Б1). Навантаження на зразки прикладалося у вигляді зосередженої сили i додавалося рівномірними ступенями 3 витримкою в 10-15 хвилин. Розміри балок $100 \times 200 \times 1200$ мм 3 величиною робочого прогону 1000 мм. У балках попередньо закладені штучні пошкодження стиснутої зони бетону різних розмірів 3 різним кутом щодо грані балки, а також використовуються різні величини відносного прольоту зрізу $(1 d, 2 d, i 3 d)$. Для балок були використані матеріали: бетон - класу C25/30, робоча поздовжня арматура - Ø 18 класу А500С, конструктивна поздовжня і поперечна арматура у вигляді хомутів - Ø 6 класу А240С. Фізико-механічні властивості матеріалів були визначені за допомогою контрольних зразків - кубів, призм і арматурних стержнів згідно діючих нормативних вимог. Всі зразки зруйнувались по похилому перерізі. Встановлено, що збільшення відносного прольоту зрізу (від $l d$ до $3 d$ ) призводить до суттєвого зменшення несучої здатності (особливо від $1 d$ до $2 d$ ). Також в межах відносного прольоту зрізу утворення пошкоджень і збільшення їх об'єму призводить до зменшення несучої здатності зразків. Отримані в результаті
\end{abstract}


експериментальних досліджень дані будуть використані при розробці методики по визначенню залишкової несучої здатності похилих перерізів.

Ключові слова: експериментальні дослідження, залізобетонні балки, пошкодження залізобетону, руйнування, похилий переріз, залишкова несуча здатність, прогін зрізу.

\title{
О РЕЗУЛЬТАТАХ ЭКСПЕРИМЕНТАЛЬНОГО ИССЛЕДОВАНИЯ ОСТАТОЧНОЙ НЕСУЩЕЙ СПОСОБНОСТИ НАКЛОННЫХ СЕЧЕНИЙ ПОВРЕЖДЕННЫХ ЖЕЛЕЗОБЕТОННЫХ БАЛОК
}

\author{
Клименко Е.В., Д.Т.н., профессор, \\ klimenkoew57@gmail.com, ORCID: 0000000245028504 \\ Бояджи А.А., к.т.н., ст. преподаватель, \\ Полянский К. В., аспирант \\ Одесская государственная академия строительства и архитектуры \\ kostyapolyanski@gmail.com
}

\begin{abstract}
Аннотация. В статье представлены результаты проведенных экспериментальных исследований по определению влияния повреждений сжатой зоны бетона на остаточную несущую способность наклонных сечений железобетонных балок прямоугольного сечения. Для проведения эксперимента была использована универсальная силовая установка и гидравлический домкрат. В соответствии с планом эксперимента, было испытано 16 однопролетных свободно опертых экспериментальных образцов-балок. Все образцы имели свой шифр от Б0 до Б15 (образец Б0 двойник образца Б1). Нагрузка на образцы прикладывалась в виде сосредоточенной силы и прибавлялась равномерными ступенями с выдержкой в 10-15 минут. Размеры балок $100 \times 200 \times 1200$ мм с величиной рабочего пролета 1000 мм. В балках предварительно заложены искусственные повреждения сжатой зоны бетона разных размеров с разным углом относительно грани балки, а также используются разные величины относительного пролета среза $(1 d, 2 d$, и $3 d)$. Для балок были использованы материалы: бетон - класса C25/30, рабочая продольная арматура - Ø 18 класса А500С, конструктивная продольная и поперечная арматура в виде хомутов - Ø 6 класса A240C. Физико-механические свойства материалов были определены с помощью контрольных образцов - кубов, призм и арматурных стержней согласно действующих нормативных требований. Все образцы разрушились по наклонному сечению. Установлено, что увеличение относительного пролета среза (от $1 d$ до $3 d$ ) приводит к существенному уменьшению несущей способности (особенно от $1 d$ до $2 d$ ). Так же в пределах относительного пролета среза образование повреждений и увеличение их объема приводит к уменьшению несущей способности образцов. Полученные в результате экспериментальных исследований данные будут использованы при разработке методики по определению остаточной несущей способности наклонных сечений.
\end{abstract}

Ключевые слова: экспериментальные исследования, железобетонные балки, повреждения железобетона, разрушение, наклонное сечение, остаточная несущая способность, пролет среза.

Стаття надійшла 3.05.2019 